\title{
Chain formation in homogeneous gas-liquid nucleation of polar fluids
}

\author{
Pieter Rein ten Wolde ${ }^{\text {a) }}$ \\ FOM Institute for Atomic and Molecular Physics, Kruislaan 407, 1098 SJ Amsterdam, The Netherlands \\ David W. Oxtoby \\ The James Franck Institute, The University of Chicago, Chicago, Illinois 60637 \\ Daan Frenkel ${ }^{\text {b) }}$ \\ FOM Institute for Atomic and Molecular Physics, Kruislaan 407, 1098 SJ Amsterdam, The Netherlands
}

(Received 10 March 1999; accepted 15 June 1999)

\begin{abstract}
We report a computer-simulation study of homogeneous gas-liquid nucleation in a model for strongly polar fluids. We find that the nucleation process is initiated by chain-like clusters. As the cluster size is increased, the chains become longer. However, beyond a certain size, the nuclei collapse to form compact, spherical clusters. Nevertheless, in the interface of the collapsed nuclei a high degree of chain formation is preserved. We compare the interface of the collapsed nuclei with the planar interface and find that the interface of the globule-like nuclei differs markedly from the flat interface. Classical nucleation theory underestimates both the size of the critical nucleus and the height of the nucleation barrier. (C) 1999 American Institute of Physics. [S0021-9606(99)50534-5]
\end{abstract}

\section{INTRODUCTION}

When simple fluids, such as the Lennard-Jones fluid, are cooled below a critical temperature $T_{c}$, they can occur in two distinct disordered phases that only differ in density: the (low-density) vapor phase and the (denser) liquid phase (see, e.g., Ref. 1). The explanation of the coexistence of both phases dates back to the work of van der Waals, who showed that it arises from the interplay of the short-range repulsive forces and the long-range attractive forces between the particles. $^{2}$

However, even if particles have both repulsive and attractive interactions, the existence of a liquid-vapor transition is not inevitable. For instance, if the range of the attractive forces is sufficiently short, the liquid-vapor transition will be pre-empted by a freezing transition..$^{3-7}$ More recently, evidence has accumulated that in a system of dipolar hard spheres (hard spheres with embedded permanent dipoles), where the attractive interactions are long-ranged, the liquidvapor transition may be suppressed. In fact, early theoretical studies of the phase behavior of this prototype dipolar fluid suggested that these systems should have a gas-liquid critical point. ${ }^{8-10}$ This seemed plausible because, in the isotropic fluid phase, the dipole-dipole interaction provides a net $r^{-6}$ attraction between the particles, where $r$ is the interparticle separation. However, when the phase behavior of dipolar hard spheres was investigated by computer simulation, no gas-liquid phase coexistence was observed. ${ }^{11,12}$ Of particular interest is a computer simulation study by Van Leeuwen and Smit. ${ }^{13}$ Van Leeuwen and Smit studied the equilibrium phase behavior of a Stockmayer fluid, in which the particles

\footnotetext{
${ }^{a}$ Present address: Department of Chemistry, University of California, Berkeley, CA 94720.

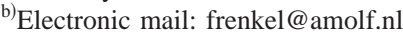

interact via a Lennard-Jones potential plus a dipole-dipole interaction potential

$$
\begin{aligned}
\mathbf{V}\left(\mathbf{r}_{i j}, \boldsymbol{\mu}_{i}, \boldsymbol{\mu}_{j}\right)= & 4 \epsilon\left[\left(\frac{\sigma}{r_{i j}}\right)^{12}-\left(\frac{\sigma}{r_{i j}}\right)^{6}\right] \\
& -3\left(\boldsymbol{\mu}_{i} \cdot \mathbf{r}_{i j}\right)\left(\boldsymbol{\mu}_{j} \cdot \mathbf{r}_{i j}\right) / r_{i j}^{5}+\boldsymbol{\mu}_{i} \cdot \boldsymbol{\mu}_{j} / r_{i j}^{3} .
\end{aligned}
$$

Here, $\epsilon$ is the Lennard-Jones well depth, $\sigma$ is the LennardJones diameter, $\boldsymbol{\mu}_{i}$ denotes the dipole moment of particle $i$, and $\mathbf{r}_{i j}$ is the vector joining particles $i$ and $j$.

The simulations of Van Leeuwen and Smit suggest that a minimum amount of dispersive attractions is required to observe gas-liquid phase coexistence. ${ }^{13}$ If the attractive forces are too small in comparison to the strength of the dipolar interactions, as for soft sphere ${ }^{14}$ and hard sphere dipolar fluids, ${ }^{11,12}$ then the system appears unable to condense to form a liquid, but appears to form a "gel" of chains of dipoles that align heat-to-tail. Theoretical studies by Van Roij ${ }^{15}$ and by Sear ${ }^{16}$ suggest that the tendency to form chains can suppress the gas-liquid phase coexistence.

In the present paper, we wish to investigate whether this peculiar feature of dipolar interactions may affect the nucleation behavior of polar fluids, even in the case where the liquid-vapor transition itself is still possible. The reason why we look at nucleation is the following: since the work of Ostwald, it is known that the phase that forms during homogeneous nucleation need not be the one that is thermodynamically most stable. ${ }^{17}$ In recent computer studies, we found evidence that even when Ostwald's rule does not apply on a macroscopic scale (i.e., at the level of the macroscopic phases that form), it may apply on a more microscopic scale to the nuclei of the new phase that forms during homogeneous nucleation. ${ }^{18-20}$

We have studied by computer simulation the pathway for condensation of a strongly polar Stockmayer fluid. The 
reduced dipole moment was chosen to be $\mu^{*}=\mu / \sqrt{\epsilon \sigma^{3}}$ $=4$, which is, in fact, comparable to that of water. For this value of the dipole moment, the system is known to show gas-liquid phase coexistence. ${ }^{21}$ However, as we will show below, the nucleation of liquid droplets is preceded by the formation of chains. Only when the chains have reached a certain size do they collapse to form compact clusters. For polar fluids without attractive dispersion forces, the tendency to form chains will be even more pronounced. It is conceivable that the gel-like phases observed in the simulations of Refs. 11 and 13 is an interpenetrating network of uncollapsed dipolar chain clusters.

The rest of this paper is organized as follows. We first present a new approach to determine the size distribution of liquid clusters in a vapor. In Sec. III we give the computational details of the simulations and we end with a discussion of the results. Parts of the results have been published elsewhere. ${ }^{22}$

\section{DETERMINATION OF CLUSTER-SIZE DISTRIBUTION}

In homogeneous gas-liquid nucleation, the density is usually so low that without association the gas would be ideal. However, the temperature is low enough for particles to associate into dimers, trimers, etc. Still, the concentration of $n$-mers is so low that we can safely ignore their mutual interactions. We thus have an ideal "solution" of $n$-mers in the vapor phase.

The identification of a cluster is not unambiguous and can only be performed explicitly after choosing a criterion that must be fulfilled by the particles that constitute an $n$-mer. We have adopted a geometric cluster criterion. All particles that are within a cutoff distance $q_{c}=1.5 \sigma$ from each other are considered to be "connected," and, therefore, belong to the same cluster. Thus, the cluster criterion $w_{n}\left(\mathbf{r}^{\prime n-1}\right)$ is given by $w_{n}\left(\mathbf{r}^{\prime n-1}\right)=1$, if all $n$ particles are mutually connected;

$=0$, otherwise.

Now that we have specified our cluster criterion, we can define the partition function $Z_{n}$ of an $n$-mer (Ref. 23) as

$$
Z_{n}=\frac{V n^{3}}{\nu^{n} n !} \int d \mathbf{r}^{\prime n-1} w_{n}\left(\mathbf{r}^{\prime n-1}\right) \exp \left[-\beta U_{n}\left(\mathbf{r}^{\prime n-1}\right)\right],
$$

where $\beta \equiv 1 / k_{B} T$ is the reciprocal temperature, with $k_{B}$ Boltzmann's constant and $T$ the absolute temperature, $V$ is the total volume of the system, $\mathbf{r}^{\prime n-1}$ denotes the coordinates of the particles in the cluster, with the prime indicating that the coordinates are taken with respect to the center-of-mass of the cluster, $U_{n}\left(\mathbf{r}^{\prime n-1}\right)$ is the interaction energy, $w_{n}\left(\mathbf{r}^{\prime n-1}\right)$ is the cluster criterion, and $\nu$ is the thermal volume

$$
\nu=\Lambda^{3} \times \frac{h^{3}}{\sqrt{\left(2 \pi k_{B} T\right)^{3} I_{1} I_{2} I_{3}}},
$$

where $\Lambda$ is the thermal de Broglie wavelength and $I_{1}, I_{2}, I_{3}$ are the principal moments of inertia of the particles.

We can define the free energy of an $n$-mer as

$$
F_{n} \equiv-k_{B} T \ln Z_{n}=-k_{B} T \ln (V / \nu) Z_{n, \mathrm{~cm}},
$$

where $Z_{n, \mathrm{~cm}}$ is the partition function for a cluster in its center-of-mass frame. Note that whereas $Z_{n, \mathrm{~cm}}$ does not depend on the size of the system (provided of course that the spatial extent of the cluster is significantly smaller than the system), $Z_{n}$, and therefore the free energy $F_{n}$, do depend on the size of the system, as they contain a contribution from the translation of the center-of-mass of the cluster over the volume $V$.

As the interactions between the clusters are neglected, the number of clusters $N_{n}$ is given by (see Refs. 23, 24)

$N_{n}=Z_{n} \exp [\beta \mu n]=\exp \left[-\beta\left(F_{n}-n \mu\right)\right] \equiv \exp [-\beta \Delta F]$,

where $\mu$ is the imposed chemical potential. The free-energy difference $\Delta F$ is the free-energy change associated with assembling $n$ particles in a cluster. Note that the magnitude of the free-energy difference depends on the size of the system as the free energy of an $n$-mer, $F_{n}$, contains a free-energy contribution from the translational free energy of the centerof-mass of the cluster. In particular, $N_{n}$, the number of clusters of size $n$, is an extensive quantity, i.e., it is proportional to the size of the system, as can be seen by combining Eq. (4) with Eq. (7). We would like to obtain a quantity that does not depend on the size of the system. We therefore define an intensive probability $P(n)$,

$$
P(n) \equiv N_{n} / N,
$$

that relates the average number $N_{n}$ of clusters of size $n$ to the total number of particles $N$ in the system. The free-energy $\Omega$ of a cluster is defined as

$$
\beta \Delta \Omega(n, \mu, V, T) \equiv-\ln [P(n)] .
$$

We stress that, in our simulations, we do not directly compute $\beta \Delta \Omega(n)$. Rather, we measure the cluster-size probability distribution function $P(n)$, as given in Eq. (8), and obtain the free energy $\beta \Delta \Omega(n)$ by taking (minus) the logarithm of this distribution function. However, in order to compute the absolute nucleation rate, we only need to know $P(n)$ itself $-\Delta \Omega(n)$ is only a derived quantity. From the cluster-size probability distribution function, $P(n)$, we directly obtain one crucial ingredient to calculate the nucleation rate: the number of nuclei at the top of the barrier. The other ingredient, which is the flux over the top of the barrier, can be obtained using molecular dynamics, as shown in Ref. 25 . In the present study, we only calculate the cluster-size probability distribution and not the full nucleation rate, i.e., we do not compute the kinetic prefactor.

The cluster-size probability distribution function $P(n)$ is an equilibrium quantity and can be measured both by Monte Carlo (MC) and molecular dynamics (MD). In principle, one could measure $P(n)$ simply by simulating a metastable vapor and counting the number of clusters. However, at moderate supersaturations, only small $n$-mers will be formed that have a free energy $\Omega$ on the order of a couple of $k_{B} T$. But, critical nuclei, that is nuclei at the top of the free-energy barrier separating the stable liquid from the metastable vapor, have a free energy on the order of $50-75 k_{B} T$. Hence, the probability that such critical nuclei will be formed spon- 
taneously is extremely small. To obtain good statistics for all cluster sizes, we have therefore used the umbrella sampling technique. ${ }^{26}$ The main idea is to bias the sampling of configuration space and correct for the bias afterwards. We can bias the sampling of configuration space by adding a fictitious potential that is a function of an order parameter to the true potential of our model system. As explained in detail in Ref. 27, the use of a global order parameter, such as the density of the system or the total number of liquid particles, has some serious drawbacks from a computational point of view. If the volume is large, it will always be entropically favorable to distribute a given amount of the new phase over many small clusters, rather than over one relatively large liquid cluster. In fact, if the volume is sufficiently large, the change in free energy associated with a small homogeneous density fluctuation will be smaller than the change in free energy due to the formation of a liquid-like droplet. However, for the nucleation process, we are interested in the largest liquid cluster that grows to its critical size. We therefore exploit the fact that the clusters can be decoupled from the surrounding vapor and simulate only one cluster in the grand canonical ensemble. The order parameter is taken to be the size of this cluster.

Kusaka et al. ${ }^{28}$ showed how the size distribution of a cluster can be obtained in the grand canonical ensemble. In their scheme, all particles in the simulation cell are considered to be part of the same cluster. Consequently, all density fluctuations in the system participate in the nucleation event. This implies that, in order to avoid redundant counting of configuration space, the fluctuations of the center-of-mass of the cluster with respect to the simulation cell should be analyzed carefully. ${ }^{29}$ Moreover, the size of the volume of the system has to be chosen carefully. On the one hand, the volume should be larger than the spatial extent of the physical cluster in it. On the other hand, as discussed above, the volume should also not be too large. If the volume is too large, the cluster will break up into many small clusters (see Ref. 27).

The use of a geometric cluster criterion not only provides a unique definition of the cluster, but also circumvents this problem. For large system sizes, it ensures that the particles that make up the cluster are always connected. But more importantly, it is not even required that the volume into (from) which the particles are inserted (removed) is larger than the size of the cluster. This is particularly important when the cluster is not compact but ramified. In the scheme by Kusaka ${ }^{28}$ and in the approach of Lee, Barker, and Abraham, ${ }^{30}$ it is conceivable that the constraining sphere biases the shape of the nucleus. In contrast, our scheme does not impose a certain cluster shape.

In order to see this, consider the combined system shown in Fig. 1. In our grand canonical MC scheme, we only consider particle additions to and removals from a spherical volume $V_{s}$ around the center-of-mass of the cluster. The centerof-mass is computed for the cluster excluding the particle to be added or removed. The acceptance probability for the insertion (removal) of particles into (from) a sphere of volume $V_{s}$ placed in a system of volume $V$ is given by ${ }^{31}$

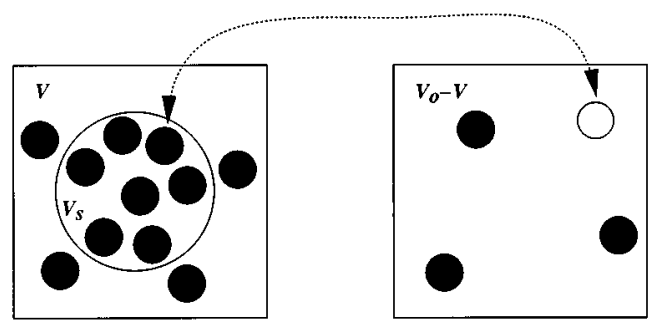

FIG. 1. Ideal gas $\left(N_{0}-N\right.$ particles, volume $\left.V_{0}-V\right)$ can exchange particles with an $N$-particle system of volume $V$. The particles are only removed/ inserted from/into a sphere of volume $V_{s}$, containing $M$ of the $N$-interacting particles.

$$
\begin{aligned}
\alpha(n \rightarrow n+1)= & \frac{V_{s} w\left(\mathbf{r}^{n+1}\right)}{(M+1) w\left(\mathbf{r}^{n}\right)} \exp [\beta \mu] \\
& \times \exp \left[-\beta\left[U\left(\mathbf{r}^{n+1}\right)-U\left(\mathbf{r}^{n}\right)\right]\right], \\
\alpha(n+1 \rightarrow n)= & \frac{(M+1) w\left(\mathbf{r}^{n}\right)}{V_{s} w\left(\mathbf{r}^{n+1}\right)} \exp [-\beta \mu] \\
& \times \exp \left[-\beta\left[U\left(\mathbf{r}^{n}\right)-U\left(\mathbf{r}^{n+1}\right)\right]\right] .
\end{aligned}
$$

Note that in the above expressions, the number of particles $M$ in the sphere appears, but not the total number of particles $n$ in the cluster. Still, the potential energy $U$ contains not only contributions from the interactions between particles inside the sphere, but also from interactions between particles inand outside the sphere. Note also that only the volume of the sphere enters the expression and not the total volume of the system. The cluster criterion is included in the acceptance criterion to ensure that the cluster remains connected.

\section{COMPUTATIONAL DETAILS}

\section{A. Nucleation barrier}

We have computed the cluster-size distribution via the grand canonical scheme discussed in Sec. II. However, rather than checking for the connectivity of the particles in the cluster at every trial move, we adopted a staged scheme in which we only checked for the connectivity of the cluster at fixed intervals.

In the first stage, a series of Monte Carlo cycles is performed. In each MC cycle, we try to both displace particles and insert or remove particles. In one cycle, on average every particle is given one trial displacement and the choice between the trial insertion/removal moves and the trial displacement moves is made at random, with $60 \%$ probability for the latter. The trial displacements are accepted with the usual Metropolis acceptance criterion. That is, we do not check whether the connectivity of the cluster is broken. Only in the second stage, after this sequence of typically 5-10 MC cycles, will we check whether all particles are still connected to one another. If all particles are not mutually connected with each other, we reject the entire sequence.

When a particle is to be inserted, we first determine the center-of-mass of the cluster. The particle is then inserted at a random position inside a sphere centered at the center-ofmass of the cluster. The addition of a particle is accepted with a probability 


$$
\begin{aligned}
& \operatorname{acc}(n \rightarrow n+1) \\
& =\min \left[1, \frac{V_{s}}{M+1} \exp [\beta \mu] \exp \left[-\beta\left[U\left(\mathbf{s}^{n+1}\right)+W(n+1)\right.\right.\right. \\
& \left.\left.\left.\quad-U\left(\mathbf{s}^{n}\right)-W(n)\right]\right]\right]
\end{aligned}
$$

where $W(n)$ is the biasing potential. The biasing potential was taken to be a harmonic function of the cluster size $n$

$$
W(n)=\frac{1}{2} k_{n}\left(n-n_{0}\right)^{2} .
$$

When a particle has to be removed, we first randomly select the particle to be removed. In order to preserve the symmetry of the underlying Markov chain, we then determine the center-of-mass of all particles except the particle to be removed and check whether the selected particle is inside the sphere centered at the center-of-mass. If the particle is outside the sphere, we repeat this procedure, until we have found a particle which is inside the sphere. The removal of the particle is then accepted with a probability

$$
\begin{aligned}
& \operatorname{acc}(n \rightarrow n-1) \\
& =\min \left[1, \frac{M}{V_{s}} \exp [-\beta \mu] \exp \left[-\beta\left[U\left(\mathbf{s}^{n-1}\right)+W(n-1)\right.\right.\right. \\
& \left.\left.\left.\quad-U\left(\mathbf{s}^{n}\right)-W(n)\right]\right]\right] .
\end{aligned}
$$

The height of the barrier and the structure of the nuclei should not depend on the size of the sphere. In particular, the sphere need not contain the entire cluster. We have verified that the results are indeed insensitive to the size of the sphere. However, the size of the sphere does affect the efficiency of the simulation. If the sphere is large, the insertion and removal probability, given by Eqs. (12) and (15), are relatively large. However, the probability that the connectivity of the cluster is broken after the Monte Carlo sequence will also be increased, and thus the probability that the trajectory has to be rejected. For smaller spheres the balance is reversed. In addition, if the sphere is very small, it can happen that the sphere does not contain any particle to be removed. We found that, depending on the shape and size of the clusters, a sphere of radius $5-10 \sigma$ is optimal.

The number of umbrella windows for the free-energy barrier was 25. Most simulations in a window consisted of an equilibration period of $5 \times 10^{5}$ cycles, followed by a production run of $5 \times 10^{5}-2 \times 10^{6}$ cycles. Especially for the smaller clusters, very long production runs were required as they exhibit strong shape fluctuations. The results that we report here are free of hysteresis.

\section{B. Coexistence point}

The coexistence point was determined by calculating the chemical potential as a function of pressure for both the vapor and liquid phase. The chemical potential and pressure at coexistence was then found from the intersection.

In order to obtain for the vapor phase the chemical potential as a function of pressure, we first determined for a vapor at low pressure $P_{0}$ (so low that association of mono- mers does not occur), the chemical potential by the Widom insertion technique. ${ }^{32}$ The chemical potential $\mu$ as a function of pressure was then obtained by integrating the inverse density $\rho$ as a function of pressure $P$

$$
\mu(P)=\mu\left(P_{0}\right)+\int_{P_{0}}^{P} \frac{1}{\rho\left(P^{\prime}\right)} d P^{\prime} .
$$

For the liquid phase we have to follow a different procedure, as the strong first-order phase transition separating the liquid from the vapor phase rules out the integration along the isotherm from a low-density vapor. We therefore exploited the fact that the liquid-vapor critical point of the Lennard-Jones system $\left(T_{c}=1.316 \epsilon / k_{B}\right)^{33}$ is much lower than that of the Stockmayer system $\left(T_{c}=5.07 \epsilon / k_{B}^{21}\right)$. We first computed the chemical potential for a Lennard-Jones system at a reference density $\rho_{0}=0.8$ by Widom insertion at low density and integration along the equation of state [see Eq. (17)]. After we have obtained the chemical potential for the Lennard-Jones system at $\rho_{0}$, we can determine the chemical potential for the Stockmayer system at this density by computing the reversible work to switch on the dipolar interactions.

In order to find a reversible path from the Lennard-Jones system to the Stockmayer system, we have used the following interaction potential:

$$
\mathrm{v}(\lambda)=\mathrm{v}_{\mathrm{LJ}}+\lambda \mathrm{v}_{\mathrm{dip}}
$$

For $\lambda=1$, the above interaction potential is given by Eq. (1), whereas for $\lambda=0$ the interaction potential reduces to the Lennard-Jones potential. The chemical potential $\mu(\lambda$ $=1, \rho_{0}$ ) of the Stockmayer system is now related to the chemical potential $\mu\left(\lambda=0, \rho_{0}\right)$ of the Lennard-Jones system via

$$
\begin{aligned}
\mu\left(\lambda=1, \rho_{0}\right)= & \mu\left(\lambda=0, \rho_{0}\right)+\int_{0}^{1}\left\langle\mathrm{v}_{\mathrm{dip}}\right\rangle_{\lambda} d \lambda \\
& +\frac{1}{\rho_{0}}[P(\lambda=1)-P(\lambda=0)] .
\end{aligned}
$$

Once we know the chemical potential of the Stockmayer system at a given density $\rho_{0}$ (and pressure $P_{0}$ ), we can obtain the chemical potential as a function of pressure by integrating along the equation of state [see Eq. (17)].

All simulations of the bulk phases were performed using a system size of 256 particles. The simulations to compute equations of state were performed in the isothermal-isobaric (NPT) ensemble, whereas the simulations to calculate the free-energy difference between the Stockmayer system and the Lennard-Jones system were done in the canonical (NVT) ensemble. In the simulations, the Lennard-Jones potential was truncated at half the box size and the standard longrange corrections were added. ${ }^{34}$ The long-range dipolar interactions were handled with the Ewald summation technique using "conducting", boundary conditions. ${ }^{35}$ Cubic periodic boundary conditions were applied. 


\section{Surface tension}

The surface tension was computed from a direct MC simulation of the two coexisting phase, using the Ewaldsummation technique to handle the long-range dipolar interactions. A liquid slab was brought in contact with a vapor, from which the surface tension $\gamma$ was obtained by measuring

$$
\gamma=\frac{1}{2} L_{z}\left(P_{z z}-\frac{1}{2}\left(P_{x x}+P_{y y}\right)\right) .
$$

Here, $P_{\alpha \alpha}$ is the $\alpha \alpha$ element of the pressure tensor and $L_{z}$ is the length of the system in the direction perpendicular to the interface. The factor of $1 / 2$ outside the bracket arises from the fact that we have two liquid-vapor interfaces in the system. In the Appendix, we describe how we have computed the pressure tensor.

In order for the density and pressure to approach bulk liquid values in the middle of the slab, the slab should not be too thin. We therefore used a rectangular simulation box, which allows for a relatively thick slab in comparison to the total number of particles in the system. The sides were of length $L_{x}=L_{y}=1 / 4 L_{z}$, for the $x$-, $y$-, and $z$-direction, respectively, and periodic boundary conditions were applied in all three coordinate directions. The maximum number of reciprocal lattice vectors parallel to the interface was $\left|k_{x}^{\max }\right|$ $=\left|k_{y}^{\max }\right|=7$, whereas the number of wave vectors perpendicular to the interface was $\left|k_{z}^{\max }\right|=28$. The larger number of reciprocal vectors in the $z$-direction makes the simulations significantly longer, but this is unavoidable in order to achieve the same convergence of the Ewald sum in all principal directions.

The Lennard-Jones interaction potential was truncated at $r_{c}=0.5 L_{x}$ and the tail correction to the surface tension was evaluated from ${ }^{36}$

$\gamma_{\text {tail }}=12 \pi \epsilon \int_{-\infty}^{\infty} \int_{-1}^{1} \int_{r_{c}}^{\infty} \rho\left(z_{1}\right) \rho\left(z_{2}\right)\left(1-3 s^{2}\right) r^{-4} d r d s d z_{1}$,

where $s=\left(z_{1}-z_{2}\right) / r$ and $z_{1}$ and $z_{2}$ are the positions of particles 1 and 2, respectively. The density profile was fitted to a hyperbolic tangent function of the form

$$
\rho(z)=\frac{1}{2}\left(\rho_{l}+\rho_{\mathrm{v}}\right)-\frac{1}{2}\left(\rho_{l}-\rho_{\mathrm{v}}\right) \tanh \left[\left(z-z_{0}\right) / d\right],
$$

where $\rho_{l}$ and $\rho_{\mathrm{v}}$ are the densities of the liquid and vapor respectively, and $z_{0}$ and $d$ are parameters for the location of the dividing surface and the thickness of the surface. With the above fit for the density profile, the tail correction becomes $^{36}$

$\gamma_{\text {tail }}=12 \pi \epsilon\left(\rho_{l}-\rho_{\mathrm{v}}\right)^{2} \int_{0}^{1} d s \int_{r_{c}}^{\infty} d r \operatorname{coth}(r s / d)\left(3 s^{3}-s\right) r^{-3}$.

In order to establish that our system is in equilibrium, we also studied the real-space contribution to the normal and tangential components of the pressure tensor as a function of $z$. When the system is in equilibrium, the normal component should be equal to the transverse component away from the interfaces.

For an inhomogeneous fluid, there is no unambiguous way to calculate the components of the pressure tensor. We have used the Irving-Kirkwood convention. ${ }^{37}$ The system is

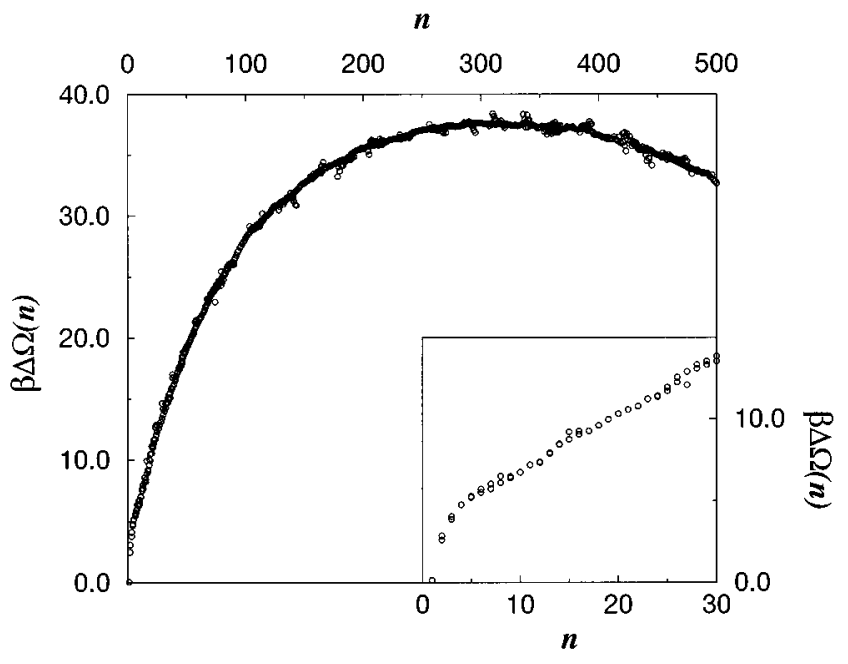

FIG. 2. The excess free energy $\beta \Delta \Omega$ of a cluster as a function of its size $n$, for a Stockmayer system at $S=1.26$ and $T^{*}=k_{B} T / \epsilon=3.5$. The reduced dipole moment $\mu^{*}=\mu / \sqrt{\epsilon \sigma^{3}}=4$.

divided into $N_{s}$ slabs parallel to the interface. The local normal $\left[p_{N}(s)\right]$ and tangential $p_{T}(s)$ components of the pressure tensor are given by

$$
p_{N}(s)=k_{B} T\langle\rho(s)\rangle+\frac{1}{V_{s}}\left\langle\sum_{i, j} s \frac{z_{i j}^{2}}{r_{i j}} f\left(\mathbf{r}_{i j}, \boldsymbol{\mu}_{i}, \boldsymbol{\mu}_{j}\right)\right\rangle
$$

and

$$
p_{T}(s)=k_{B} T\langle\rho(s)\rangle+\frac{1}{2 V_{s}}\left\langle\sum_{i, j} s \frac{x_{i j}^{2}+y_{i j}^{2}}{r_{i j}} f\left(\mathbf{r}_{i j}, \boldsymbol{\mu}_{i}, \boldsymbol{\mu}_{j}\right)\right\rangle,
$$

where $\rho(s)$ is the average density in slab $s, V_{s}$ $=L_{x} L_{y} L_{z} / N_{s}$ is the volume of the slab and $f\left(\mathbf{r}_{i j}, \boldsymbol{\mu}_{i}, \boldsymbol{\mu}_{j}\right)$ $=-\left(\partial \mathbf{v}\left(\mathbf{r}_{i j}, \boldsymbol{\mu}_{i}, \boldsymbol{\mu}_{j}\right) / \partial r_{i j}\right)$ is the force acting between particles $i$ and $j . \Sigma_{i j}^{s}$ denotes a summation that runs over all pairs of particles $i$ and $j$ for which the slab $s$ (partially) contains the line that connects them. The contribution to the virial of a slab from a given pair is determined by the ratio to which the slab contains the line.

We performed a simulation with 512 particles and one with 2000 particles, to check for finite-size effects. The number of equilibration cycles was 100000 and the number of production cycles was 200000 .

\section{RESULTS AND DISCUSSION}

All simulations were performed at $T^{*}=k_{B} T / \epsilon=3.5$ (with $k_{B}$ Boltzmann's constant), which is approximately $30 \%$ below the critical temperature of $T_{c}^{*}=5.07 .{ }^{21}$ We first study the formation of a critical nucleus at an imposed chemical potential $\mu=-26.0 \epsilon$. This corresponds to a supersaturation $S=\left(P / P_{\text {coex }} \approx \exp [\beta \Delta \mu]\right)=1.26$, where $\Delta \mu=\mu$ $-\mu_{\text {coex }}$, with $\mu(P)$ the imposed chemical potential (pressure), and $\mu_{\text {coex }}\left(P_{\text {coex }}\right)$ the chemical potential (pressure) at coexistence. Figure 2 shows the excess free energy of a cluster as a function of its size at this degree of supersaturation.

From the free-energy barrier measured at this degree of supersaturation, we can directly obtain the nucleation barrier 
at any degree of supersaturation, as long as the interactions between the clusters can be neglected. In that case, the partition function $Z_{n}$ of an $n$-mer is independent of fugacity. That is, the configuration integral of $Z_{n}$ in Eq. (4) does not depend on the imposed chemical potential. We can then combine Eqs. (4) and (7) with Eq. (9) to arrive at

$$
\begin{aligned}
\beta \Delta \Omega\left(n, \mu^{\prime}, V, T\right)= & \beta \Delta \Omega(n, \mu, V, T)-\beta\left(\mu^{\prime}-\mu\right) n \\
& +\ln \left[\rho\left(\mu^{\prime}\right) / \rho(\mu)\right],
\end{aligned}
$$

where $\rho=N / V$ is the total number density in the system. We have verified that our simulations indeed satisfy this equation by computing the free-energy barriers for two different chemical potentials. Furthermore, we have compared the structure of the nuclei at one chemical potential with that at the other chemical potential. The analysis of the structural order parameters, which will be discussed below, indicates that the nucleus structure is independent of the imposed fugacity. This provides further evidence that the sampling of configuration space and hence the evaluation of the configuration integral in Eq. (4) does not depend on the chemical potential. We have therefore determined the free-energy barrier as a function of supersaturation from the computed freeenergy barrier at $S=1.26$, using Eq. (26).

As the structure of the clusters does not depend on the imposed fugacity, we only discuss the structure analysis performed at $S=1.26$. After the structure analysis, we will consider how the nucleus structure determines the free-energy barrier. We compare the free-energy barrier, as well as the critical nucleus size, with the corresponding predictions of classical nucleation theory. However, in order to compare the structure of the nuclei with that of the bulk liquid, we first investigate the structural order in the liquid.

\section{A. Equilibrium phase behavior}

Stevens and Grest found evidence for the existence of dipolar order in the fluid phase, albeit for different conditions (i.e., higher temperature and pressure). ${ }^{21} \mathrm{We}$ therefore checked whether the bulk liquid also exhibits ferroelectric ordering at the temperature and range of pressures at which we study the nucleation process.

Ferroelectric ordering can be characterized by the order parameter $P_{1}$, which is defined as

$$
P_{1}=\frac{1}{N}\left|\sum_{i=1}^{N} \hat{\boldsymbol{\mu}}_{i} \cdot \hat{\mathbf{d}}\right|=\frac{1}{\mu N}|\mathbf{M} \cdot \hat{\mathbf{d}}|,
$$

where $\hat{\boldsymbol{\mu}}_{i}$ is a unit vector specifying the orientation of the dipole of particle $i, \mathbf{M}$ is the total dipole moment of the system, and $\hat{\mathbf{d}}$ is the director, which is the eigenvector corresponding to the largest eigenvalue of the $\mathbf{Q}$-tensor

$$
\mathbf{Q}=\frac{1}{N} \sum_{i=1}^{N}\left(\frac{3}{2} \hat{\boldsymbol{\mu}}_{i} \hat{\boldsymbol{\mu}}_{i}-\frac{\mathbf{I}}{2}\right) .
$$

For a fully ordered system, $P_{1}=1$. We found that $P_{1}$ did not differ significantly from zero for the bulk liquid at the temperature and supersaturations considered in the simulations. Hence, the system does not show significant ferroelectric ordering.

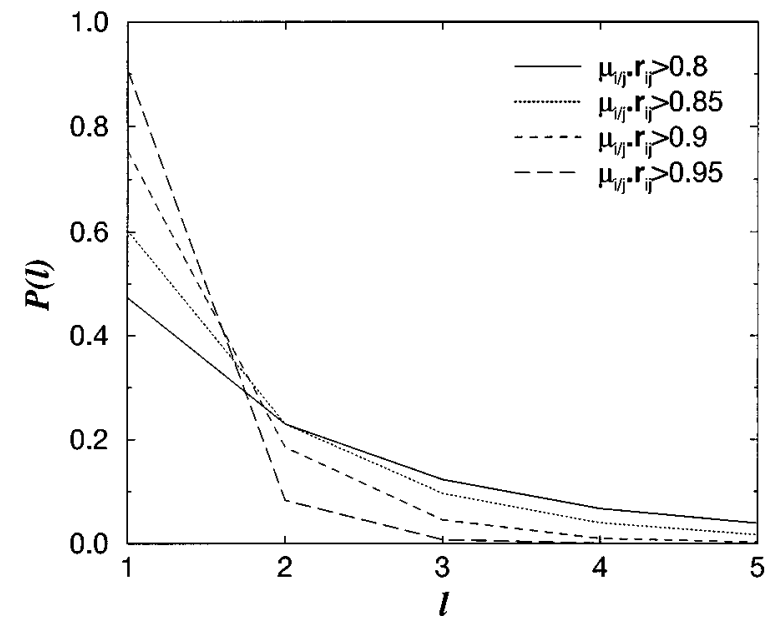

FIG. 3. Probability distribution functions of the chain-length $l$ in a thermally equilibrated bulk liquid at a supersaturation of $S=1.76\left(T^{*}=3.5\right.$ and $P^{*}$ $=0.005)$. The different curves correspond to different threshold values of the chain criterion (see the text).

Yet, it is conceivable that in the bulk liquid a high degree of chain formation is present. We therefore examined the chain-size distribution. We have adopted the following chain criterion: particles $i$ and $j$ are part of the same chain if the interparticle distance $r_{i j}$ is less than $q_{c}$ and for both particles the dot product $\hat{\boldsymbol{\mu}}_{i / j} \cdot \hat{\mathbf{r}}_{i j}$ exceeds a certain threshold. Figure 3 shows the chain-size distribution for different threshold values. As expected, the size distribution is slightly sensitive to the value of the threshold. Still, it is clear that most chains consist of only one to four particles and that chain formation is not very pronounced. We therefore conclude that the bulk liquid at the temperature and range of pressures considered in the simulations is isotropic. In order to avoid any confusion, we have only used the chain criterion to identify the chains in the liquid. As mentioned in Sec. II, we have used a distance criterion to identify the particles that make up a cluster in the vapor.

\section{B. Coil-globule transition}

In classical nucleation theory it is assumed that even the smallest droplets are spherical. In fact, this is a reasonable approximation for a typical model system for nonpolar fluids, the Lennard-Jones system. In Ref. 23 we showed that precritical nuclei as small as ten particles are already quite compact, spherical objects. However, the interaction potential of the Lennard-Jones system is isotropic, whereas the dipolar interaction potential is anisotropic. It is possible that this affects the shape of the small clusters. Indeed, for bulk hard-sphere and soft-sphere dipolar fluids at low temperature, it has been observed that particles associate into chains. ${ }^{11,14}$ On the other hand, as discussed in the previous section, the bulk liquid is isotropic.

We find that clusters containing up to 30 particles form chains in which the particles align head-to-tail. In fact, we find a whole variety of differently shaped clusters in dynamical equilibrium with each other: linear chains, branched chains, and ring-"polymers." As the cluster size is increased, the polymers become longer. But, beyond a certain 


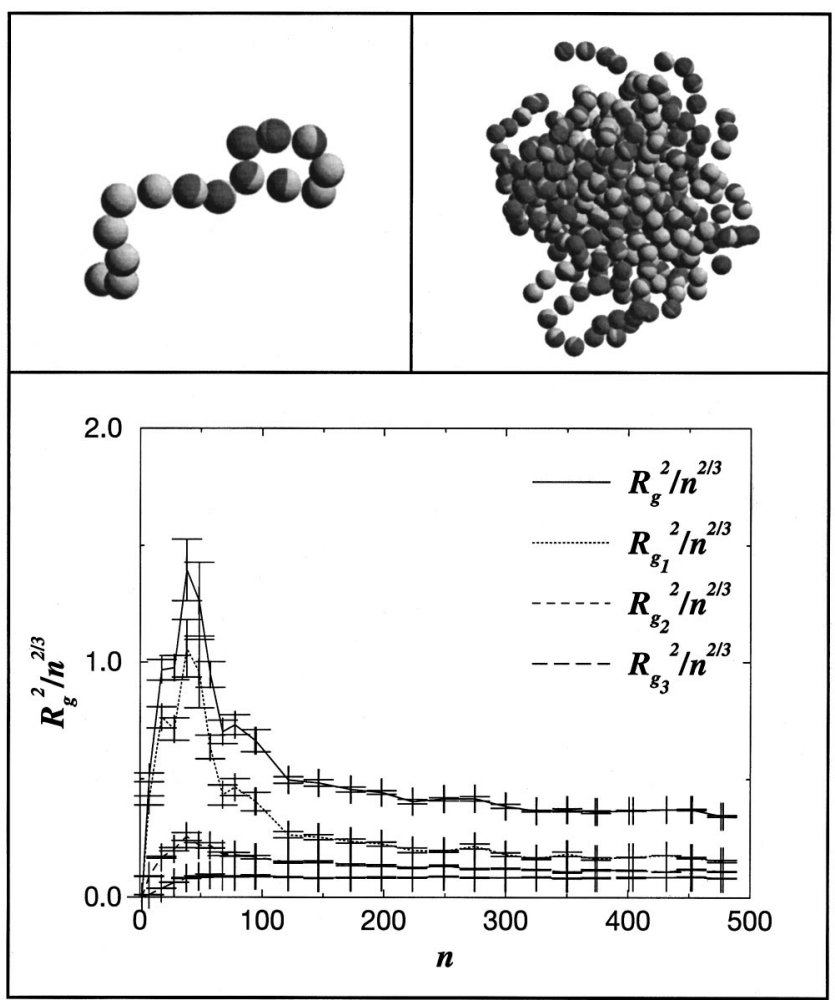

FIG. 4. Radius of gyration $R_{g}$, and the three eigenvalues of the momentof-inertia tensor, as a function of cluster size $n$, at $S=1.26$ and $T^{*}=3.5$. Initially, the clusters are chain-like (snapshot top left), but at a cluster size of $n \approx 30$ they collapse to compact, spherical nuclei (snapshot top right).

size, the clusters collapse to form a compact globule. In order to quantify this, we determined the size dependence of the radius of gyration, as well as the three eigenvalues of the moment-of-inertia tensor I

$$
\mathbf{I}=\frac{1}{n} \sum_{i=1}^{n} \mathbf{r}_{i} \mathbf{r}_{i}
$$

where $\mathbf{r}_{i}$ is the vector joining particle $i$ and the center-ofmass of the cluster. In Fig. 4, we show the square of the radius of gyration, divided by $n^{2 / 3}$. For a compact, spherical object $R_{g}^{2}$ scales with $n^{2 / 3}$, whereas for chains $R_{g}^{2}$ scales with $n^{\alpha}$, where $1.2<\alpha<2$, depending on the stiffness of the chain. Hence, for chain-like clusters $R_{g}^{2} / n^{2 / 3}$ should increase with $n$, whereas for a globule it should approach a constant value.

Figure 4 shows that initially $R_{g}^{2} / n^{2 / 3}$ increases with the size of the cluster. Moreover, one eigenvalue of the moment of the inertia matrix is much larger than the other two, which indicates the strong tendency of clusters to form chains. However, at a cluster size of about 30 particles, $R_{g}^{2} / n^{2 / 3}$ starts to decrease and approaches a constant value at a cluster size of around 200 particles. Furthermore, at that point the three eigenvalues of the moment-of-inertia tensor have also approached each other: the cluster has collapsed to a compact spherical object.

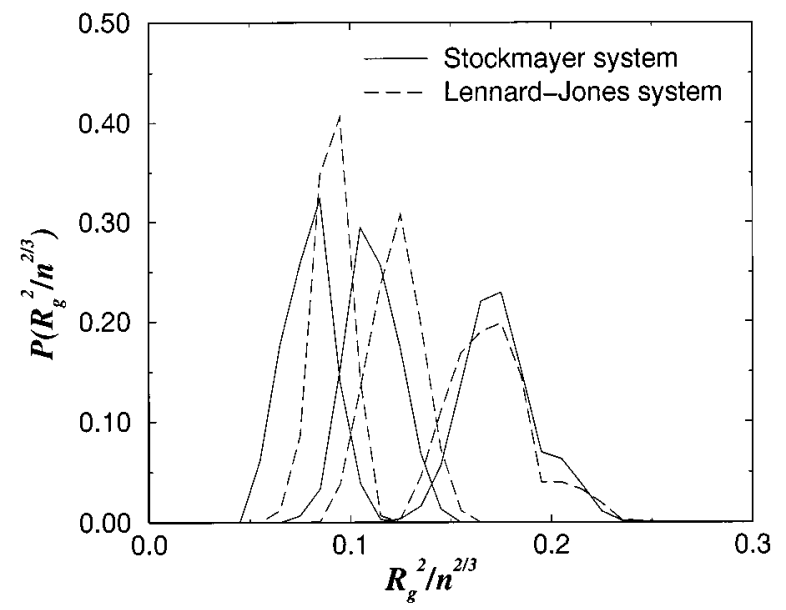

FIG. 5. The distribution functions of the three eigenvalues of the momentof-inertia tensor for a cluster of around 300 particles in a Stockmayer system at $T^{*}=3.5\left(=0.7 T_{c}\right)$ and $S=1.26$. For comparison, we also show the distribution functions for a Lennard-Jones cluster of comparable size $(n \approx 300)$ and at the same temperature with respect to the critical temperature ( $T$ $=0.7 T_{c}$ ). In this and subsequent figures, $\sigma$ is the unit of length.

\section{Structure of collapsed nuclei}

\section{No global order}

An examination of the snapshots of the larger, globulelike nuclei confirms that they are compact, more or less spherical objects. To study this in more detail, we have measured the probability distribution functions of the three eigenvalues of the moment-of-inertia tensor. Figure 5 shows the result. For comparison, we also indicate the distribution functions for a Lennard-Jones cluster of comparable size (and at the same temperature with respect to the critical temperature). Note that compared to the Stockmayer cluster, the distribution functions of the Lennard-Jones cluster are displaced to somewhat higher values. This is due to the slightly lower density in the Lennard-Jones cluster. Yet, for both the Lennard-Jones and the Stockmayer systems the distribution functions of the three eigenvalues overlap. Hence, the clusters are quite spherical, although the larger spread of the distribution functions of the polar cluster indicates that this cluster exhibits more pronounced fluctuations around the spherical shape. We do not find evidence for a prolateshaped cluster, in which the molecules are aligned in an antiparallel head-to-tail arrangement, as suggested by Wright and El-Shall. ${ }^{38,39}$ A prolate structure would have two small eigenvalues and one significantly larger eigenvalue of the moment-of-inertia tensor. As can be seen from Fig. 5, this is not the case. Furthermore, both the nematic order parameter $P_{2}$, which is the largest eigenvalue of the $\mathbf{Q}$ tensor defined in Eq. (29), and the ferroelectric order parameter $P_{1}$ [see Eq. (28)] are zero, indicating that there is no net parallel or antiparallel alignment of the dipoles in the cluster.

We also investigated the degree of circulating orientational order. To this end, we computed the order parameter

$$
\mu_{11}=\left|\boldsymbol{\mu}_{11}\right|=\left(\sum_{\alpha=x, y, z} \mu_{11 \alpha}^{2}\right)^{1 / 2},
$$

with 


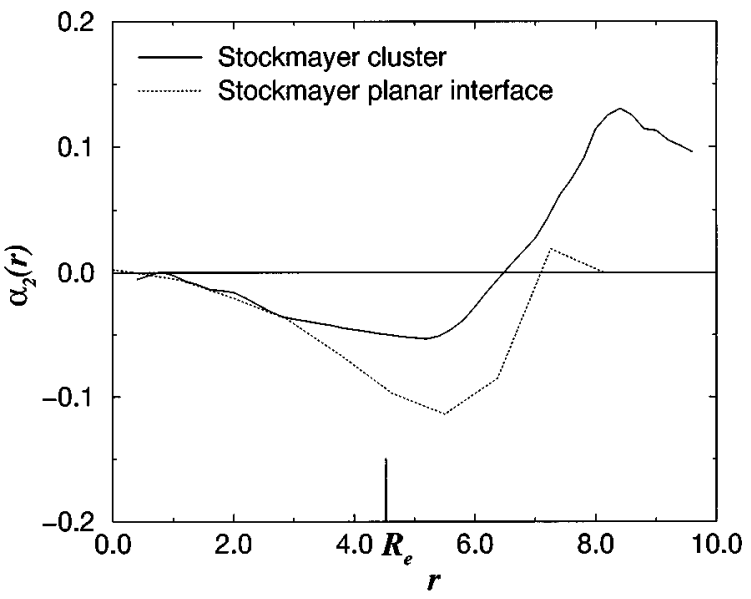

FIG. 6. The profile of the orientational order parameter $a_{2}$, as defined in Eq. (33), for a planar interface at coexistence and for a cluster of approximately 300 particles at a supersaturation $S=1.26$. The profile of the planar interface is shifted such that its equimolar dividing surface coincides with that of the cluster, as indicated by the vertical line at $R_{e}$.

$$
\mu_{11 \alpha}=\frac{1}{n} \sum_{i=1}^{n}\left(\hat{\mathbf{e}}_{\alpha} \times \hat{\mathbf{r}}_{i}\right) \cdot \hat{\boldsymbol{\mu}}_{i},
$$

where $\hat{\mathbf{e}}_{\alpha}$ is a unit vector in the $\alpha$-direction, $\hat{\boldsymbol{\mu}}_{i}$ denotes the orientation of the dipole of particle $i$, and $\hat{\mathbf{r}}_{i}$ is the unit vector from the center-of-mass of the cluster to particle $i$. This order parameter was introduced by Singer et $a l^{40}$ and measures the degree to which the cluster contains dipoles circulating about a particular axis. Singer et al. ${ }^{41}$ have studied Stockmayer clusters consisting of 50 particles with a reduced dipole moment of $\mu^{*}=\sqrt{3}$, and found that the (liquid) clusters had an oblate shape with a large degree of circulating orientational order up to $T^{*}=0.8$, which is $60 \%$ below $T_{c}^{*}$. However, this temperature is much lower than in the present simulations $\left(T^{*}=0.7 T_{c}^{*}\right)$, and indeed we do not find evidence for an oblate shape (one eigenvalue of the moment-of-inertia tensor would then be significantly smaller than the other two), nor do we find any significant circulating orientational order $\left(\mu_{11}<0.14\right)$. Hence, the global order parameters indicate that the large, collapsed clusters are spherical with no net global orientational order.

\section{Interfacial structure}

In the previous section, we found that the larger, collapsed nuclei are spherical, with no net global orientational order. Let us next examine the local order. Given the spherical shape of the clusters, it is meaningful to analyze the local order as a function of the distance $r$ to the center-of-mass of the cluster. Figure 6 shows the radial profile of $\alpha_{2}$, which is defined as

$$
\alpha_{2}=\sqrt{5 / 4 \pi}\left\langle P_{2}(\cos \theta)\right\rangle,
$$

where $\theta$ is the angle between the director of the dipole and the normal to the surface. It measures the degree to which the dipoles are oriented perpendicular to the surface. It is seen that $\alpha_{2}$ approaches zero in the center of the droplet. This indicates that the orientation of the dipoles in the core of the droplets is isotropic and suggests that the core of the droplet

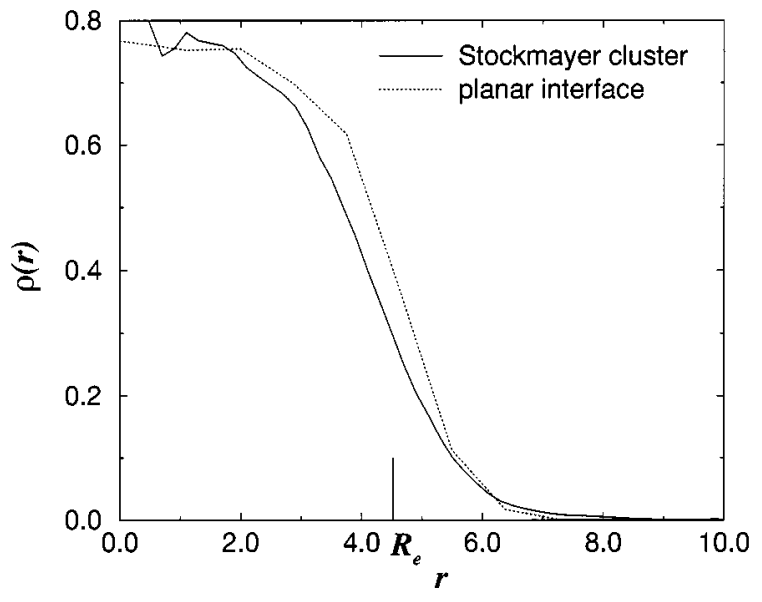

FIG. 7. Density profile for a planar vapor-liquid interface at coexistence and for a cluster of around 300 particles at a supersaturation $S=1.26\left(T^{*}\right.$ $=3.5$ ). The density profile of the planar interface is shifted such that the equimolar dividing surface of the planar interface coincides with the equimolar dividing surface of the cluster. The equimolar dividing surface is indicated by the vertical line at $R_{e}$.

shows bulk liquid behavior. To verify this we have measured the density profile, which is shown in Fig. 7. It is also seen that the density approaches a bulk liquid value in the core of the droplets. It thus seems that the core of the larger, collapsed nuclei is characterized by bulk liquid properties.

However, in the interface the nuclei start to lose their bulk liquid character. Figure 6 shows that near the interface the orientation of the dipoles is not isotropic. In agreement with previous simulation results ${ }^{41,42}$ and theoretical studies, ${ }^{43}$ we find that at the liquid (core) side of the interface the dipoles tend to orient parallel to the interface, whereas at the vapor side they prefer a perpendicular orientation. Hence, the assumption that the dipoles are oriented perpendicular to the surface at the liquid side of the interface ${ }^{44}$ is not justified for this polar fluid.

In most nucleation theories, it is assumed that the surface tension of a cluster is that of a planar interface at coexistence. It is therefore natural to compare the structure of the interface of the nuclei with that of the flat interface. Figure 6 shows the comparison for the $\alpha_{2}$-profile. It is also seen that for the planar interface the dipoles tend to align parallel to the interface at the liquid side, but perpendicular to the interface at the vapor side. However, the ordering is much more pronounced for the planar interface at the liquid side. In contrast, at the vapor side the ordering is stronger for the cluster interface.

Still, both for the planar interface and the interface of the nuclei, $\alpha_{2}$ changes sign after the equimolar dividing surface. In this respect, our findings are in agreement with those of Singer et $a l .{ }^{41}$ However, the simulation results contradict the results of a density functional study of homogeneous nucleation in a (weakly) polar fluid, in which it was found that both for the planar interface and for the cluster interface, $\alpha_{2}$ changes sign before the equimolar dividing surface. ${ }^{43}$

In our study of crystal nucleation in a Lennard-Jones system, we found that the structure of the small clusters, which initiate the nucleation process, still persists in the in- 


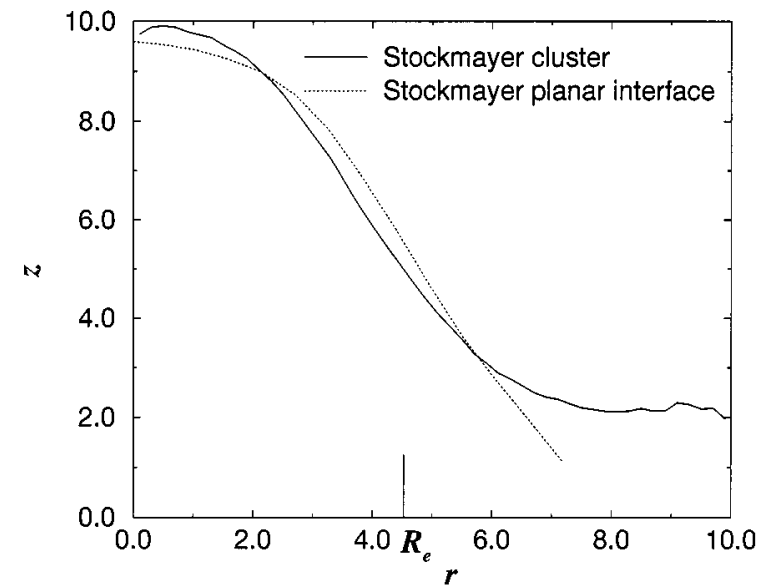

FIG. 8. Radial profile of the coordination number for a Stockmayer cluster at $S=1.26$ and $T^{*}=3.5$ (solid line). The coordination number $z$ is defined as the number of particles within a cutoff distance $q_{c}=1.5 \sigma$. For comparison, we also give the coordination number profile for a planar interface (dotted curve), which is shifted in such a way that its equimolar dividing surface coincides with that of the cluster, which is indicated by the vertical line at $R_{e}$.

terface of the larger clusters. To be more precise, we observed that the structure in the core is that of the thermodynamically most stable phase, but that the core is "wetted" by a shell with a structure characteristic for the smaller clusters. We have therefore examined whether in the present case we also find traces of the tendency to form chains at the surface of the larger, collapsed, clusters.

A visual inspection (see Fig. 4) of these clusters suggests that loops of dipolar chains stick out of the surface (leading to a positive value of $\alpha_{2}$, see Fig. 6). In order to quantify this behavior, we have computed the radial profile of the coordination number for a cluster of around 300 particles. This is shown in Fig. 8. It is seen that the coordination number smoothly approaches a value of 2 at the vapor side of the interface. Such behavior is expected if the particles on the vapor side of the interface belong to chains. For comparison, we have also shown in Fig. 8 the coordination number profile for the planar interface. As the number density in the vapor is extremely low, smaller than $1 \times 10^{-3} \sigma^{-3}$, it was not possible to get accurate statistics beyond $r=6.75 \sigma$. However, it is clearly seen that at the vapor side of the planar interface the coordination number drops below 2 . Hence, the interface of the clusters is more rough than the planar interface. Also, an inspection of the density profiles (see Fig. 7) shows that the planar interface is sharper than the interface of a collapsed cluster of around 300 particles - the width of the interface of the cluster is some $25 \%$ larger. Most likely, the planar interface is sharper because the stronger dispersion interactions with the bulk cause the dipole chains to adsorb.

\section{Comparison with classical nucleation theory}

Classical nucleation theory (CNT) is based on macroscopic thermodynamics. It is clear that the use of macroscopic quantities has both its advantages and disadvantages. However, even if we accept the approximations of classical nucleation theory, there are still inconsistencies within the theory. In 1961, Courtney argued that the CNT prediction for the cluster distribution did not satisfy the law of mass action, ${ }^{45}$ and suggested that this problem could be resolved by introducing the factor $1 / S$, where $S$ is the supersaturation, into the expression for the cluster distribution. Although the factor $1 / S$ does bring CNT into line with the law of mass action, its introduction seems rather ad hoc. ${ }^{46}$ Moreover, Lothe and Pound realized that a more fundamental omission in the original theory still persists. ${ }^{47}$ They argued that classical nucleation theory does not properly take into account the mechanical and rotational degrees of freedom of a cluster. This is now often referred to as the problem of the "replacement free energy" and is related to the problem of the redundant counting of configuration space, as mentioned in Sec. II. The above issues have been the subject of controversy, although recently much progress has been made. ${ }^{29}$

In classical nucleation theory (CNT), the excess free energy of a cluster as a function of its size is given by

$$
\beta \Delta \Omega(n)=\beta\left[\left(\frac{36 \pi}{\rho_{l}^{2}}\right)^{1 / 3} \gamma n^{2 / 3}-\Delta \mu n\right],
$$

where $\rho_{l}$ is the density of the bulk liquid (which is assumed to be incompressible), $\gamma$ is the surface tension of a planar vapor-liquid interface at coexistence, and $\Delta \mu=\mu_{\mathrm{v}}\left(P_{\mathrm{v}}\right)$ $-\mu_{l}\left(P_{\mathrm{v}}\right)$ is the difference in chemical potential between a bulk liquid and a bulk vapor, both at the imposed pressure $P_{\mathrm{v}}$.

The prediction of classical nucleation theory for the height of the nucleation barrier and the size of the critical nucleus can be obtained by taking the derivative of Eq. (34) with respect to the cluster size $n$. We then find for the height of the barrier

$$
\beta \Delta \Omega^{*}=\beta \frac{16 \pi \gamma^{3}}{3 \rho_{l}^{2} \Delta \mu^{2}},
$$

and for the size of the critical nucleus

$$
n^{*}=\frac{32 \pi \gamma^{3}}{3 \rho_{l}^{2} \Delta \mu^{3}} .
$$

In order to compare our simulation results with classical nucleation theory, we computed $\gamma$ by the procedure outlined in Sec. III C. We found $\gamma=1.34 \pm 0.07 \epsilon / \sigma^{2}$. As the density in the vapor is much lower than the density in the liquid, the $\mu(P)$ curve of the vapor phase is much steeper than that of the liquid. We therefore made the common assumption that the difference in chemical potential can be approximated by $\Delta \mu=\mu_{\mathrm{V}}\left(P_{\mathrm{V}}\right)-\mu_{l}\left(P_{\mathrm{V}}\right) \simeq \mu_{\mathrm{v}}\left(P_{\mathrm{V}}\right)-\mu_{\text {coex }}$. The chemical potential at coexistence was determined using the method discussed in Sec. III B and was found to be $\mu_{\text {coex }}=-26.82$ $\pm 0.02 \epsilon$. The density of the liquid at this chemical potential is $\rho_{l}=0.78 \pm 0.01 \sigma^{-3}$.

Let us now discuss in more detail the basic assumptions of classical nucleation theory that lead to Eqs. (34)-(36). First, it is assumed that the nuclei behave like small droplets of bulk liquid that are spherical. As discussed previously, the larger nuclei, comprising more than approximately 200 particles, are quite spherical. However, the smaller clusters, which initiate the nucleation process in this system, are not 

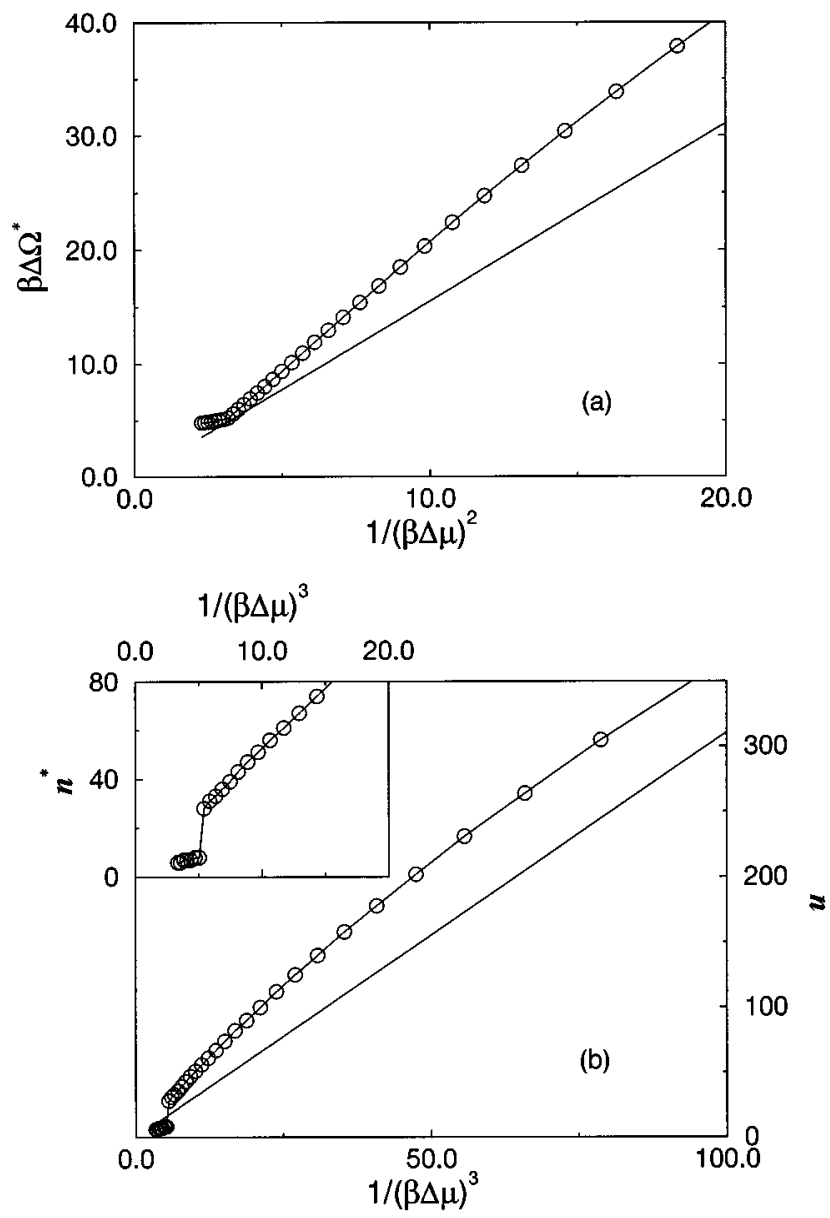

FIG. 9. Comparison of the simulation results (open circles) with classical nucleation theory (straight solid line) for a Stockmayer system at $S=1.26$ and $T^{*}=3.5$ : (a) nucleation barrier; (b) size of the critical cluster.

compact, spherical objects, but chain-like aggregates. As a consequence, the variation in free energy with cluster size is not as predicted by classical nucleation theory. We note that if the clusters were perfectly rigid chains, the variation in free energy with cluster size would be linear. Indeed, as can be seen from Fig. 2, we find that after $n \approx 5$ the increase in cluster free energy is very linear with its size. The barrier shape predicted by classical nucleation theory [see Eq. (34)] is only recovered after the clusters have collapsed.

The linear regime in the size dependence of the cluster free energy has a remarkable consequence for the dependence of the critical nucleus size on supersaturation [see Fig. 9(a)]. When the supersaturation is increased, the height of the nucleation barrier and the critical nucleus size decrease. However, at a supersaturation of $S=1.8\left(1 /(\beta \Delta \mu)^{3}=5.25\right)$, the critical clusters have reached a size at which the variation in free energy with cluster size is linear. At this point, the top of the barrier is flat, and a small increase in the supersaturation leads to a jump in the critical cluster size [see inset of Fig. 9(b)].

It is thus clear that at large supersaturation, where the critical nuclei are relatively small, the polymer-like character of the clusters leads to strongly nonclassical nucleation behavior. However, even for the collapsed globule-like nuclei, the simulation results show large deviations from classical nucleation theory. This can be seen from Fig. 9. This figure shows the comparison between the simulation results and the predictions of classical nucleation theory for the height of the barrier and the critical nucleus size. Clearly, the theory underestimates both the size of the critical nucleus and the height of the nucleation barrier. As the variation in the nucleation rate is dominated by the variation in the barrier height, our results are in qualitative agreement with the experiments on strongly polar fluids, ${ }^{38,39}$ in which it was found that classical nucleation theory seriously overestimates the nucleation rate.

We would like to understand the origin of this discrepancy between classical nucleation theory and the simulations. As mentioned, the larger, collapsed nuclei are spherical. Also, the assumption of classical nucleation theory that the cores of these nuclei show bulk liquid behavior seems to be justified, as the density and the orientational order parameter $\alpha_{2}$ approach bulk liquid values in the center of the droplets.

However, CNT neglects the variation of surface tension with droplet size. In fact, McGraw and Laaksonen ${ }^{48}$ showed that the interfacial curvature free energy can cause a significant correction to the barrier height as predicted by classical nucleation theory. They derived relations ${ }^{48,49}$ for the barrier height and the critical nucleus size that could provide an explanation for the discrepancy between experiment and classical nucleation theory for a variety of nonpolar and weakly polar fluids. ${ }^{50}$ In particular, McGraw and Laaksonen ${ }^{48}$ showed that if CNT correctly predicts the size of the critical nucleus, then the curvature correction yields a constant offset between the actual barrier height and the height of the barrier as predicted by classical nucleation theory. It is interesting to note that this behavior has been observed in both a density functional study ${ }^{48,49}$ and in our computer simulation study of gas-liquid nucleation in the Lennard-Jones system (see Ref. 23). It was found that for a wide range of cluster sizes, CNT gives a correct prediction of the critical nucleus size and that the deviation from the predicted barrier height is constant, i.e., independent of cluster size.

However, for this polar system, the displacement between the measured barrier height and the predicted height of the barrier is not constant, as can be seen from Fig. 9. More clearly, the critical nucleus size is not correctly predicted by CNT. Hence, in contrast to the (nonpolar) Lennard-Jones fluid, the relations as proposed by McGraw and Laaksonen ${ }^{48,49}$ are not obeyed for this highly polar fluid. A possible explanation could be that, whereas the interface of a Lennard-Jones critical nucleus is nearly indistinguishable from the planar interface, for this system the structure of the interface of the critical clusters is still markedly different from the planar interface.

\section{CONCLUSIONS}

We have studied homogeneous gas-liquid nucleation of a strongly polar Stockmayer fluid. The simulations show that the nucleation process is initiated by chain-like clusters. When these clusters exceed a certain size, they condense to form compact, droplet-like nuclei. However, the interface of 
these droplets, with a high degree of chain formation, is still markedly different from the planar interface. In highly polar liquids, ${ }^{38,39}$ it may be responsible for the discrepancy between the experimentally observed nucleation rates and the predictions of classical nucleation theory.

\section{ACKNOWLEDGMENTS}

This work was supported by Chemische Wetenschappen (CW) with financial aid from (NWO) (Nederlandse Organisatie voor Wetenschappelijk Onderzoek). The work of the FOM Institute is part of the research program of Stichting Fundamenteel Onderzoek der Materie (FOM) and is supported by NWO. David Oxtoby acknowledges support from the U.S. Nation Science Foundation (Grant. No. CHE 9800074).

\section{APPENDIX: PRESSURE TENSOR}

For pairwise additive potentials, such as the LennardJones potential, the $\alpha \beta$ element of the pressure tensor can be evaluated from the virial equation for the pressure

$$
P_{\alpha \beta}=\rho k_{B} T+\frac{1}{V} \sum_{i<j}^{N} r_{i j \alpha} f_{i j \beta},
$$

where $\mathbf{r}_{i j}$ is the vector between $i$ and $j$ and $f_{i j \beta}\left(\mathbf{r}_{i j}, \boldsymbol{\mu}_{i}, \boldsymbol{\mu}_{j}\right)$ $=-\left(\partial \mathbf{V}\left(\mathbf{r}_{i j}, \boldsymbol{\mu}_{i}, \boldsymbol{\mu}_{j}\right) / \partial r_{i j \beta}\right)$ is the intermolecular force. However, in the Ewald sum, which contains a real space contribution and a reciprocal space contribution, the pressure tensor cannot be written as in Eq. (A1).

The electrostatic energy $U_{\text {dip }}$ of the dipolar interactions in the Stockmayer system, embedded in a material with infinite dielectric constant, is ${ }^{35}$

$$
\begin{aligned}
U_{\text {dip }}= & \frac{1}{2} \sum_{i \neq j}^{N}\left(\boldsymbol{\mu}_{i} \cdot \boldsymbol{\mu}_{j}\right) B\left(r_{i j}\right)-\left(\boldsymbol{\mu}_{i} \cdot \mathbf{r}_{i j}\right)\left(\boldsymbol{\mu}_{j} \cdot \mathbf{r}_{i j}\right) C\left(r_{i j}\right) \\
& -\frac{2 \alpha^{3}}{3 \sqrt{\pi}} \sum_{i=1}^{N} \mu_{i}^{2} \\
& +\frac{2 \pi}{V} \sum_{\mathbf{k} \neq 0} Q(k) M(\mathbf{k}) M(-\mathbf{k}),
\end{aligned}
$$

where $\alpha$ is the convergence parameter,

$$
\begin{aligned}
B(r) \equiv & {\left[\frac{2 \alpha r}{\sqrt{\pi}} \exp \left(-\alpha^{2} r^{2}\right)+\operatorname{erfc}(\alpha r)\right] / r^{3}, } \\
C(r) \equiv & {\left[\frac{2 \alpha r}{\sqrt{\pi}}\left(3+2 \alpha^{2} r^{2}\right) \exp \left(-\alpha^{2} r^{2}\right)\right.} \\
& +3 \operatorname{erfc}(\alpha r)] / r^{5},
\end{aligned}
$$

with erfc the complementary error function, and

$$
Q(k) \equiv \frac{\exp \left(-k^{2} / 4 \alpha^{2}\right)}{k^{2}},
$$

and

$$
M(\mathbf{k}) \equiv \sum_{i=1}^{N} i\left(\boldsymbol{\mu}_{i} \cdot \mathbf{k}\right) \exp \left(i \mathbf{k} \cdot \mathbf{r}_{i}\right) .
$$

Here, $\mathbf{k}$ are the reciprocal lattice vectors, which are defined as

$$
\mathbf{k} \equiv\left(2 \pi l / L_{x}, 2 \pi m / L_{y}, 2 \pi n / L_{z}\right),
$$

where $l, m$, and $n$ are integers.

The first two terms in Eq. (A2) are the real-space contributions, the third term is the so-called self term expressing the self-energy of the dipoles, and the last term in Eq. (A2) is the reciprocal space contribution. The real-space contribution to the pressure tensor is obtained by evaluating the second term on the right-hand side of Eq. (A1)

$$
\begin{aligned}
P_{\alpha \beta}= & \frac{1}{2 V} \sum_{i \neq j}^{N} r_{i j \alpha}\left\{C ( r _ { i j } ) \left[r_{i j \beta}\left(\boldsymbol{\mu}_{i} \cdot \boldsymbol{\mu}_{j}\right)+\mu_{i \beta}\left(\boldsymbol{\mu}_{j} \cdot \mathbf{r}_{i j}\right)\right.\right. \\
& \left.+\mu_{j \beta}\left(\boldsymbol{\mu}_{i} \cdot \mathbf{r}_{i j}\right)\right] \\
& \left.+\frac{C^{\prime}\left(r_{i j}\right)}{r_{i j}} r_{i j \beta}\left(\boldsymbol{\mu}_{i} \cdot \mathbf{r}_{i j}\right)\left(\boldsymbol{\mu}_{j} \cdot \mathbf{r}_{i j}\right)\right\}
\end{aligned}
$$

where

$$
\begin{aligned}
C^{\prime}(r) \equiv & \frac{\partial C}{\partial r}=-\left[15 \operatorname{erfc}(\alpha r)+\frac{2 \alpha r}{\sqrt{\pi}}\left(15+10 \alpha^{2} r^{2}\right.\right. \\
& \left.\left.+4 \alpha^{4} r^{4}\right) \exp \left(-\alpha^{2} r^{2}\right)\right] / r^{6}
\end{aligned}
$$

In order to calculate the reciprocal space contribution to the pressure tensor, we have followed the procedure of Nosé and Klein. ${ }^{51}$ In this procedure, the pressure tensor is obtained from the variation in potential energy with a deformation of the system. The reciprocal space contribution to the pressure tensor is

$$
\begin{aligned}
P_{\alpha \beta}= & \frac{2 \pi}{V^{2}} \sum_{\mathbf{k} \neq 0} Q(k)\left\{M(\mathbf{k}) M(-\mathbf{k})\left(\delta_{\alpha \beta}-\frac{2 k_{\alpha} k_{\beta}}{k^{2}}-\frac{k_{\alpha} k_{\beta}}{2 \alpha^{2}}\right)\right. \\
& \left.+M(\mathbf{k}) V_{\alpha \beta}(-\mathbf{k})+M(-\mathbf{k}) V_{\alpha \beta}(\mathbf{k})\right\},
\end{aligned}
$$

where $V_{\alpha \beta}(\mathbf{k})$ is given by

$$
V_{\alpha \beta}(\mathbf{k})=\sum_{i=1}^{N} i \mu_{i \alpha} k_{\beta} \exp \left(i \mathbf{k} \cdot \mathbf{r}_{i}\right) \text {. }
$$

A similar expression has been independently derived by Heyes, ${ }^{52}$ although in Ref. 52 a minus sign in the second exponential on the right-hand side of Eq. (34) is missing.

We found that the above expressions for the pressure tensor could be used as a convergence test for the Ewald summation. From the definition of the dipolar interaction potential [see Eq. (1)] and from the definition of the virial equation for the pressure [see Eq. (A1)] it can be verified that for dipolar interactions the interaction energy is equal to the virial, i.e.,

$$
\sum_{i<j}^{N} \mathbf{v}\left(\mathbf{r}_{i j}, \boldsymbol{\mu}_{i}, \boldsymbol{\mu}_{j}\right)=\frac{1}{3} \sum_{i<j}^{N} \mathbf{r}_{i j} \cdot \mathbf{f}\left(\mathbf{r}_{i j}, \boldsymbol{\mu}_{i}, \boldsymbol{\mu}_{j}\right) .
$$




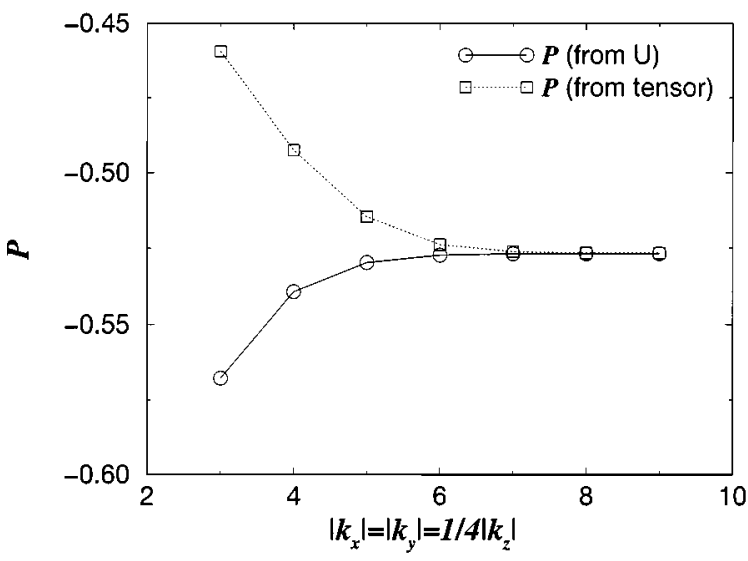

FIG. 10. Convergence test for the Ewald summation. Indicated are two ways of obtaining the pressure, as a function of the number of reciprocal lattice vectors. The line with the open circles gives the pressure obtained from the potential energy. The dotted curve with the square symbol gives the pressure obtained via the trace of the pressure tensor, i.e., via Eq. (A9) and Eq. (A12). It is seen that both routes give the same pressure after $\left|k_{x}^{\max }\right|=\left|k_{y}^{\max }\right|=1 / 4\left|k_{z}^{\max }\right|=7$.

Hence, the virial and thus the pressure can be directly obtained from the potential energy. On the other hand, the virial should also given by the trace of the stress tensor, i.e., by the sum of the traces of the stress tensors in Eqs. (A9) and (A12). In Fig. 10, we compare the two ways of obtaining the pressure. It is seen that the pressures obtained via the two routes nicely converge to the same value after $\left|k_{x}^{\max }\right|=\left|k_{y}^{\max }\right|$ $=1 / 4\left|k_{z}^{\max }\right|=7$.

${ }^{1}$ J.-P. Hansen and I. R. McDonald, Theory of Simple Liquids (Academic, London, 1990).

${ }^{2}$ J. S. Rowlinson and B. Widom, Molecular Theory of Capillarity (Clarendon, Oxford, 1982).

${ }^{3}$ A. P. Gast, W. B. Russell, and C. K. Hall, J. Colloid Interface Sci. 96, 251 (1983).

${ }^{4}$ A. P. Gast, W. B. Russell, and C. K. Hall, J. Colloid Interface Sci. 109, 161 (1986).

${ }^{5}$ H. N. W. Lekkerkerker, W. C. K. Poon, P. N. Pusey, A. Stroobants, and P. B. Warren, Europhys. Lett. 20, 559 (1992).

${ }^{6}$ M. H. J. Hagen and D. Frenkel, J. Chem. Phys. 101, 4093 (1994).

${ }^{7}$ S. M. Ilett, A. Orrock, W. C. K. Poon, and P. N. Pusey, Phys. Rev. E 51, 1344 (1995).

${ }^{8}$ P. G. de Gennes and P. A. Pincus, Phys. Kondens. Mater. 11, 189 (1970).

${ }^{9}$ M. Wertheim, J. Chem. Phys. 55, 4291 (1971).

${ }^{10}$ G. Rushbrooke, G. Stell, and J. Hoye, Mol. Phys. 26, 1199 (1973).

${ }^{11}$ J. J. Weis and D. Levesque, Phys. Rev. Lett. 71, 2729 (1993).

${ }^{12}$ J. M. Caillol, J. Chem. Phys. 98, 9835 (1993).
${ }^{13}$ M. E. van Leeuwen and B. Smit, Phys. Rev. Lett. 71, 3991 (1993).

${ }^{14}$ M. J. Stevens and G. S. Grest, Phys. Rev. Lett. 72, 3686 (1994).

${ }^{15}$ R. van Roij, Phys. Rev. Lett. 76, 3348 (1996).

${ }^{16}$ R. P. Sear, Phys. Rev. Lett. 76, 2310 (1996).

${ }^{17}$ W. Ostwald, Z. Phys. Chem., Stoechiom. Verwandtschaftsl. 22, 289 (1897).

${ }^{18}$ P. R. ten Wolde, M. J. Ruiz-Montero, and D. Frenkel, Phys. Rev. Lett. 75, 2714 (1995).

${ }^{19}$ P. R. ten Wolde, M. J. Ruiz-Montero, and D. Frenkel, J. Chem. Phys. 104, 9932 (1996).

${ }^{20}$ P. R. ten Wolde and D. Frenkel, Science 277, 1975 (1997).

${ }^{21}$ M. J. Stevens and G. S. Grest, Phys. Rev. E 51, 5976 (1995).

${ }^{22}$ P. R. ten Wolde, D. W. Oxtoby, and D. Frenkel, Phys. Rev. Lett. 81, 3695 (1998).

${ }^{23}$ P. R. ten Wolde and D. Frenkel, J. Chem. Phys. 109, 9901 (1998).

${ }^{24}$ According to Reiss et al., Eq. (7) is even valid for rare clusters that interact. See H. Reiss and G. A. Merry, J. Chem. Phys. 85, 3313 (1981).

${ }^{25}$ P. R. ten Wolde and D. Frenkel, J. Chem. Phys. 110, 1591 (1999).

${ }^{26}$ G. M. Torrie and J. P. Valleau, Chem. Phys. Lett. 28, 578 (1974).

${ }^{27}$ P. R. ten Wolde, M. J. Ruiz-Montero, and D. Frenkel, Faraday Discuss. 104, 93 (1996).

${ }^{28}$ I. Kusaka, Z.-G. Wang, and J. H. Seinfeld, J. Chem. Phys. 108, 3416 (1998).

${ }^{29}$ H. Reiss, W. K. Kegel, and J. L. Katz, J. Phys. Chem. 102, 8548 (1998).

${ }^{30}$ J. K. Lee, J. A. Barker, and F. F. Abraham, J. Chem. Phys. 58, 3166 (1973).

${ }^{31}$ A. Papadopoulou, E. D. Becker, M. Lupkowski, and F. van Swol, J. Chem. Phys. 98, 4897 (1993).

${ }^{32}$ B. Widom, J. Chem. Phys. 39, 2808 (1963).

${ }^{33}$ B. Smit, J. Chem. Phys. 96, 8639 (1992).

${ }^{34}$ D. Frenkel and B. Smit, Understanding Molecular Simulation, From Algorithms to Applications (Academic, San Diego, 1996).

${ }^{35}$ S. W. de Leeuw, J. W. de Perram, and E. R. Smith, Annu. Rev. Phys. Chem. 37, 245 (1986).

${ }^{36}$ J. Alejandre, D. J. Tildesley, and G. A. Chapela, J. Chem. Phys. 102, 4574 (1995).

${ }^{37}$ J. H. Irving and J. G. Kirkwood, J. Chem. Phys. 18, 817 (1950).

${ }^{38}$ D. Wright, R. Caldwell, C. Moxeley, and M. S. El-Shall, J. Chem. Phys. 98, 3356 (1993).

${ }^{39}$ D. Wright and M. S. El-Shall, J. Chem. Phys. 98, 3369 (1993).

${ }^{40}$ H. B. Lavender, K. A. Iyer, and S. J. Singer, J. Chem. Phys. 101, 7856 (1994).

${ }^{41}$ D. Lu and S. J. Singer, J. Chem. Phys. 103, 1913 (1995).

${ }^{42}$ A. P. Shreve, J. P. R. B. Walton, and K. E. Gubbins, J. Chem. Phys. 85, 2178 (1986).

${ }^{43}$ V. Talanquer and D. W. Oxtoby, J. Chem. Phys. 99, 4670 (1993).

${ }^{44}$ F. F. Abraham, Science 168, 833 (1970).

${ }^{45}$ W. G. Courtney, J. Chem. Phys. 35, 2249 (1961).

${ }^{46}$ C. L. Weakliem and H. Reiss, J. Chem. Phys. 101, 2398 (1994).

${ }^{47}$ J. Lothe and G. M. Pound, J. Chem. Phys. 36, 2080 (1962).

${ }^{48}$ R. McGraw and A. Laaksonen, J. Chem. Phys. 106, 5284 (1997).

${ }^{49}$ R. McGraw and A. Laaksonen, Phys. Rev. Lett. 76, 2754 (1996).

${ }^{50}$ V. Talanquer, J. Chem. Phys. 106, 9957 (1997).

${ }^{51}$ S. Nosé and M. L. Klein, Mol. Phys. 50, 1055 (1983).

${ }^{52}$ D. M. Heyes, Phys. Rev. B 49, 755 (1994). 